\title{
Educate, Nurture, Advise, Before Life Ends Comprehensive Heartcare for Patients and Caregivers (ENABLE CHF-PC): study protocol for a randomized controlled trial
}

Rachel Wells', Macy L. Stockdill', J. Nicholas Dionne-Odom', Deborah Ejem', Kathryn L. Burgio 2,3,

Raegan W. Durant ${ }^{4}$, Sally Engler ${ }^{1}$, Andres Azuero ${ }^{1}$, Salpy V. Pamboukian ${ }^{5}$, Jose Tallaj ${ }^{5}$, Keith M. Swetz ${ }^{2}$,

Elizabeth Kvale ${ }^{6}$, Rodney O. Tucker ${ }^{7}$ and Marie Bakitas ${ }^{1,2^{*}}$ (i)

\begin{abstract}
Background: Palliative care is specialized medical care for people with serious illness that is focused on providing relief from symptoms and stress and improving the quality of life (QOL) for patients and their families. To help the 6.5 million U.S. adults and families affected by heart failure manage the high symptom burden, complex decision-making, and risk of exacerbation and death, the early integration of palliative care is critical and has been recommended by numerous professional organizations. However, few trials have tested early outpatient community-based models of palliative care for patients diagnosed with advanced heart failure and their caregivers. To address this gap, through a series of formative evaluation trials, we translated an oncology early palliative care telehealth intervention for heart failure to create ENABLE CHF-PC (Educate, Nurture, Advise, Before Life Ends, Comprehensive Heartcare for Patients and Caregivers).

Methods/Design: The primary objective of this multisite pragmatic randomized controlled trial is to test the efficacy of ENABLE CHF-PC plus usual heart failure care compared to usual care alone. Community-dwelling persons who are $\geq 50$ years of age with New York Heart Association class III/IV or American Heart Association/American College of Cardiology stage C/D heart failure and their primary caregiver (if present) are being randomized to one of two study arms. The ENABLE CHF-PC intervention group receives usual heart failure care plus an in-person palliative care assessment by a board-certified palliative care provider (caregivers are invited to attend), a series of nurse coach-led, weekly psychoeducational 20 to 60 min phone sessions using a guidebook called Charting Your Course (patients: 6 sessions and caregivers: 4 sessions), and monthly check-in calls. Charting Your Course topical content includes problem-solving, coping, self-care and symptom management, communication, decision-making, advance care planning, and life review (patients only). Primary outcomes include patient QOL and mood (depressive symptoms/anxiety) and caregiver QOL, mood, and burden at 8 and 16 weeks after baseline. Outcomes will be examined using an intention-to-treat approach and mixed effects modeling for repeated measures.

(Continued on next page)
\end{abstract}

\footnotetext{
* Correspondence: mbakitas@uab.edu

${ }^{1}$ School of Nursing, University of Alabama at Birmingham, 1720 2nd Avenue South, Birmingham, AL 35294-1210, USA

${ }^{2}$ Department of Medicine, Division of Gerontology, Geriatrics, Palliative Care, University of Alabama at Birmingham, 1720 2nd Avenue South, Birmingham, AL 35294-1210, USA

Full list of author information is available at the end of the article
}

(c) The Author(s). 2018 Open Access This article is distributed under the terms of the Creative Commons Attribution 4.0 International License (http://creativecommons.org/licenses/by/4.0/), which permits unrestricted use, distribution, and reproduction in any medium, provided you give appropriate credit to the original author(s) and the source, provide a link to the Creative Commons license, and indicate if changes were made. The Creative Commons Public Domain Dedication waiver (http://creativecommons.org/publicdomain/zero/1.0/) applies to the data made available in this article, unless otherwise stated. 
(Continued from previous page)

Discussion: This trial will determine whether the ENABLE CHF-PC model of concurrent heart failure palliative care is superior to usual heart failure care alone in achieving higher patient and caregiver QOL, improving mood, and lowering burden.

Trial registration: ClinicalTrials.gov, NCT02505425. Registered on 22 July 2015.

Keywords: Palliative care, Psychoeducational intervention, Telehealth, Access

\section{Background}

Approximately 6.5 million American adults have received a diagnosis of heart failure (HF) [1]. Despite treatment advances, $22 \%$ of patients will die within 1 year of first hospitalization and close to $50 \%$ will die within 5 years [1, 2]. By 2030, it is predicted that the prevalence of HF will increase to over 8 million [3]. The morbidity and symptom burden due to HF is often high. Nearly 3 in 4 people suffer from at least one co-morbidity, the most prevalent of which include chronic kidney disease, anemia, diabetes, and chronic obstructive pulmonary disease [4]. The burden of HF is debilitating and extends beyond the patient, who often relies on close family members and friends to assist with medical management and to support their day-to-day activities of living. These tasks can become onerous over time and lead to caregivers experiencing distress and poor mental health [5].

The early integration of palliative care is critical and has been recommended in numerous organizational statements in both the U.S. and worldwide to help persons and families affected by HF manage the high symptom burden, complex decision-making, and ever-present risk of exacerbation and death, [6-15]. The World Health Organization defines palliative care as:

An approach that improves the quality of life of patients and their families facing the problem associated with life-threatening illness, through the prevention and relief of suffering by means of early identification and impeccable assessment and treatment of pain and other problems, physical, psychosocial and spiritual [16].

Palliative care is often mistakenly associated with hospice and end-of-life care [17, 18]. However, the American Heart Association and other professional organizations recommend providing palliative care concurrent with usual HF treatment [10, 15].

Despite these guideline recommendations, there has been little progress in incorporating essential palliative care services into HF care, especially early in the disease trajectory when there is the greatest potential for benefit [18-22]. The 2013 American College of Cardiology Foundation/American Heart Association (ACC/AHA) Guideline for the Management of Heart Failure recommended palliative care for all patients with a New York Heart Association (NYHA) class III/IV and ACC/AHA stage $\mathrm{C} / \mathrm{D}$. However, only one out of three HF patients receive palliative care $[15,24]$. Older patients with $\mathrm{HF}$ and their family caregivers (FCGs) rarely have access to palliative supportive care services because the disease trajectory is unpredictable and palliative treatment may not be provided until all other medical treatments have been tried.

While palliative care has been shown to be beneficial in other disease populations, such as cancer [25, 26], there have been few clinical trials to evaluate the early integration of HF palliative care in outpatient and community settings. Observational studies of hospital-based palliative care services report that palliative care consultations may reduce symptom burden and improve quality of life (QOL) [2730]. Additionally, recent reviews demonstrate decreased healthcare resource utilization with palliative care intervention, although the strongest evidence is for cancer populations [31]. While this is a positive indication of the potential benefits of palliative care, little progress has been made in incorporating these essential palliative care services into HF care. Current elements of recommended HF care management are often lacking, such as self-care, activation, decision support, communication, advance care planning, and care coordination [23, 26, 28, 32-36].

Early concurrent palliative cancer care achieves beneficial outcomes in QOL, symptom burden, depression, and in some cases, survival in oncology patients [3739]. ENABLE (Educate, Nurture, Advise, Before Life Ends) is a nurse-led multicomponent telehealth care model that provides foundational evidence supporting the World Health Organization continuum of care. It has been mentioned in a recent consensus statement [40] and a Cochrane Systematic Review [41]. The World Health Organization model posited that introducing key palliative care concepts early in the disease trajectory would increase opportunities for patients and families to benefit from all that palliative care has to offer [16]. Unlike hospice care, ENABLE is not prognosis-dependent or focused solely on end of life [42].

In our pilot study, ENABLE CHF-PC (Comprehensive Heartcare For Patients and Caregivers), instruments and procedures from previous ENABLE interventions were successfully adapted for advanced HF patients and their caregivers, thus demonstrating feasibility and acceptability of this model to a predominantly underserved HF population [42]. Therefore, the ENABLE 
CHF-PC randomized controlled trial (RCT) seeks to determine whether the ENABLE CHF-PC intervention leads to improved self-reported outcomes of QOL, mood, symptom burden, resource use, and caregiverreported health and burden.

\section{Methods/design}

Conceptual foundation

The conceptual framework of ENABLE CHF-PC is based on Wagner's Chronic Care Model (CCM). The CCM is built on collaborative coordinated care, which leads to improved outcomes by promoting self-management and decision support to foster the development of activated empowered patients $[43,44]$. Patient activation has been identified as an essential element in improving health-care quality and efficiency, and patient clinical and functional outcomes. Previous interventional studies and meta-analyses using the CCM have demonstrated improved disease-focused HF self-management and survival outcomes [45-53]. Furthermore, based on these previous successes and a study that demonstrated the successful application of the CCM with the ENABLE intervention in advanced cancer [37], ENABLE CHF-PC was successfully pilot tested for advanced HF $[42,54]$. ENABLE CHF-PC maintains the original CCM framework by fostering patient activation and empowerment [54] and effective communication about advanced care planning, treatment goals, prognosis, and symptom management [55-57] through a multicomponent coaching approach comprising an in-person palliative care consultation (PCC) [58] and telehealth patient and caregiver sessions. These components are complementary and reinforcing. The in-person PCC provides a guideline-based [58] comprehensive assessment and builds a foundation for future consultation, if needed, and the phone-based coaching sessions provide comprehensive information in an unhurried and convenient home setting. These components are standardized but tailored to individual patient and caregiver needs. The goal of ENABLE CHF-PC is to normalize the early introduction of guideline-recommended [10, 15, 21-23] palliative care content (e.g., dyspnea management, informed decision-making, etc.) by embedding palliative nurse coaches within current HF care. Thus, HF clinicians and patients are exposed to palliative care principles early while concurrently seeking diseasemodifying treatment.

\section{Aims and hypotheses}

The study aims are:

1. To determine whether ENABLE CHF-PC leads to a higher level of advanced HF patient-reported QOL and mood (depression/anxiety) [Primary Outcomes] and lower symptom burden and resource use (e.g., hospital admissions and intensive care unit days, and emergency visits) [Secondary Outcomes] at 8 and 16 weeks after baseline.

We hypothesize that intervention participants will experience a higher QOL and mood, and lower symptom burden and resource use (i.e., emergency room visits, intensive care unit days, inpatient days or hospital admissions) at 8 and 16 weeks after baseline compared with those receiving usual HF care.

2. To determine whether ENABLE CHF-PC leads to higher caregiver-reported QOL, mood (anxiety/ depression) [Primary Outcomes], and self-reported health [Secondary Outcome] and lower caregiver burden at 8 and 16 weeks after baseline.

We hypothesize that intervention caregivers will report higher QOL, mood, and self-reported health, and lower caregiver burden at 8 and 16 weeks after baseline.

The exploratory aims of the present study are also twofold:

1. To explore mediators and moderators of patient and caregiver outcomes and reciprocal relationships.

2. To examine intervention effects of using joint modeling approaches.

\section{Setting}

The study is a blinded randomized controlled clinical trial conducted at an academic tertiary referral center [University of Alabama at Birmingham (UAB)] and the Birmingham Veterans Affairs Medical Center (BVAMC).

\section{Study procedures}

Study staff target recruitment efforts around potential participants' scheduled HF clinic visits. After completing a baseline interview, patients are randomly assigned to receive usual HF care or usual HF care plus the ENABLE CHF-PC intervention. FCG participants are assigned to a group based on the patient's group allocation. The Standard Protocol Items: Recommendation for Intervention Trials (SPIRIT) checklist $[59,60]$ is included as Additional file 1. The SPIRIT diagram [59] (Fig. 1) depicts time points for enrollment and allocation activities, intervention, and data collection.

\section{Participants}

Specifically, patients meeting the eligibility criteria are recruited from the outpatient UAB HF clinic, the UAB hospital-based transitional care HF clinic, and the BVAMC HF clinic. Patient participants are not excluded 


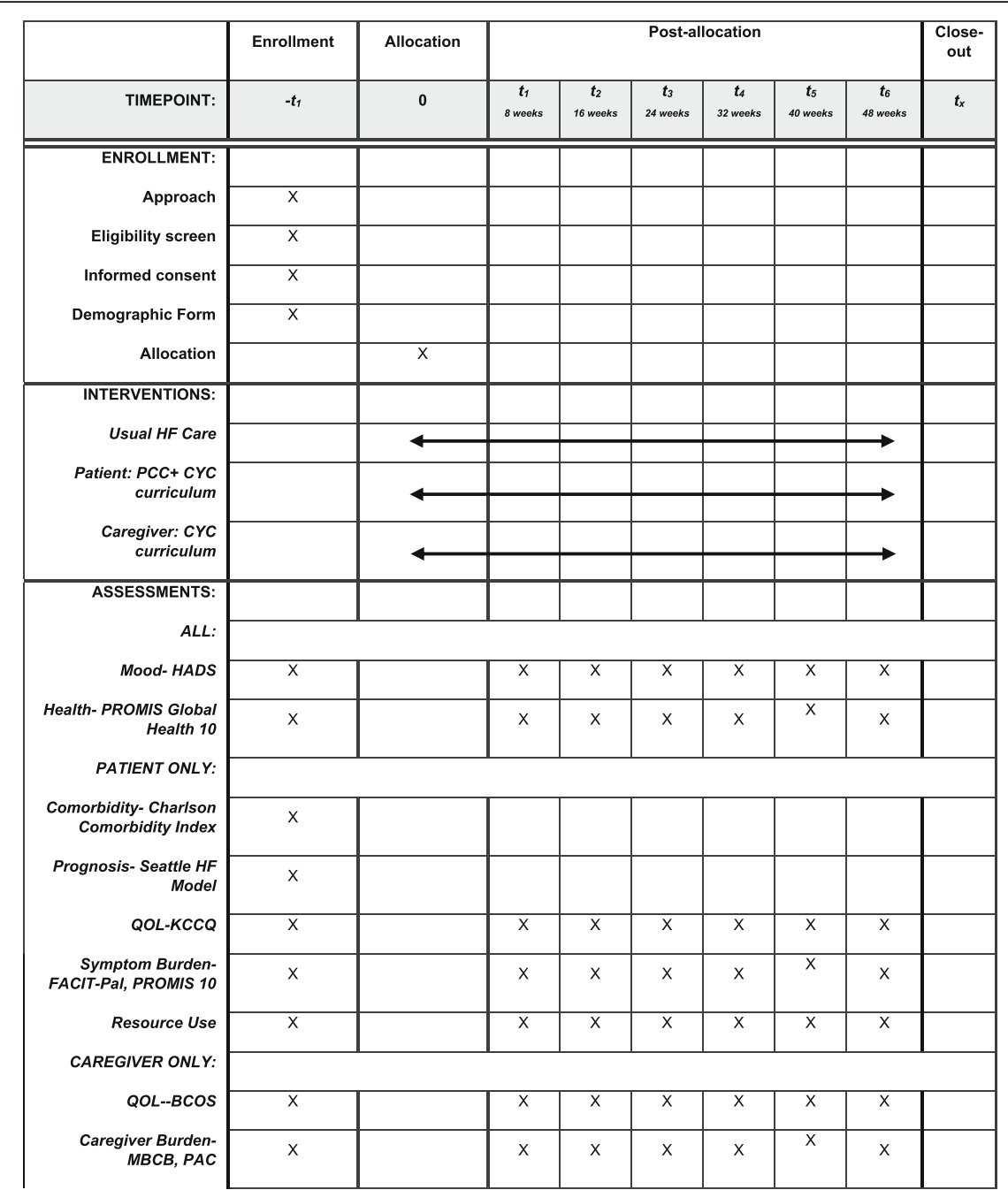

Notes: $\mathrm{HF}=$ heart failure; $\mathrm{CYC}=$ Charting Your Course; $\mathrm{HF}=$ heart failure; $\mathrm{PCC}=$ in-person palliative care consultation; HADS = Hospital Anxiety and Depression Scale; PROMIS = Patient-Completed Health Outcome Measures Global Health 10; QOL= quality of life; KCCQ= Kansas City Cardiomyopathy Questionnaire; FACIT-Pal = Functional Assessment of Chronic Illness Therapy- Palliative care; BCOS= Bakas Caregiving Outcomes Scale; $\mathrm{MBCB}=$ Montgomery Borgatta Caregiver Burden; $\mathrm{PAC}=$ Positive Aspects of Caregiving Questionnaire

Fig. 1 Evaluation of 2013 SPIRIT-recommended content in the ENABLE CHF-PC RCT. CYC Charting Your Course, HF heart failure, PCC in-person palliative care consultation, HADS Hospital Anxiety and Depression Scale, PROMIS Patient-Reported Health Outcome Measures Global Health, QOL quality of life, KCCQ Kansas City Cardiomyopathy Questionnaire, FACIT-Pal Functional Assessment of Chronic Illness Therapy: Palliative Care, BCOS Bakas Caregiving Outcomes Scale, MBCB Montgomery Borgatta Caregiver Burden, PAC Positive Aspects of Caregiving Questionnaire

if they do not identify a FCG; however, FCGs cannot enroll without concurrent patient enrollment.

Eligibility criteria for patient participants include:

1. Able to speak English

2. Age $\geq 50$ years

3. Clinician-determined NYHA class III/IV or ACC/ AHA stage C/D HF [61]

4. Reliable telephone access

5. Able to complete a baseline interview
Exclusion criteria for patient participants includes any of the following:

1. Dementia or significant confusion (measured by Callahan score $\geq 13$ ) [62]

2. Non-correctable hearing loss

3. Diagnostic and Statistical Manual of Mental Disorders (4th edition) axis I diagnosis (e.g., schizophrenia, bipolar disorder, or active substance use disorder) 
The definition of FCG used in the study is broad. FCGs may include a spouse, an adult family member, or a close friend who is considered the primary caregiver and is willing to participate [63].

Eligibility criteria for FCG participants include:

1. Identification by the patient as "a person who knows you well and is involved in your care"

2. Able to speak English

3. Age $\geq 18$ years

4. Able to complete the baseline interview

5. Reliable telephone access

\section{Recruitment}

Recruitment and data collection are conducted by a UAB community-based research and recruitment team. Team members, trained to identify advancedHF patients, screen UAB and BVAMC cardiology clinic schedules for eligible participants. Potential participants are approached after clinicians' approval during scheduled outpatient cardiology clinic appointments and eligibility is verified. If the patient agrees to participate, a written consent form is signed. During the informed consent discussion, patients are asked to identify a caregiver for study participation. If the caregivers are present during a clinic appointment, they are consented concurrently with the patients. At UAB, if the caregivers are not present, they are contacted and consented by telephone. At BVAMC, all caregiver approaches and consent must be completed in person. Recruitment began in October 2015.

\section{Randomization and blinding}

After consent is obtained, a baseline interview is completed by phone. Patients are stratified by center (UAB or BVAMC), referring service (HF clinic, cardiology, or other ), and race (Caucasian or non-Caucasian). Patients are randomized using a 1:1 ratio to either intervention or usual care. The study program manager is responsible for randomization and verifies a computer-generated treatment assignment with a randomization table. A study staff member notifies participants about their allocation status. Data collectors are blinded regarding participants' group assignment. Due to the nature of the study, participants and nurse coaches are aware of allocation to the intervention group.

\section{Data collection}

A secure REDCap (Research Electronic Data Capture) database is used to capture all data collection points. The data collection section of the database consists of three parts: (1) screened participants' contact information, (2) call attempt log, and (3) collected data measures. Trained research personnel collect patient- and caregiver-reported data by phone which is then directly entered into the REDCap database. Patient- and caregiver-reported outcome measures are collected every 8 weeks for 48 weeks. Primary outcome measures collected for patient participants include QOL and mood. Primary outcome measures collected for caregiver participants include QOL, mood, and burden. Secondary patient outcome measures collected include health status, symptom burden, and resource use. Secondary caregiver outcome measures include self-reported health. Exploratory outcomes include mediators, moderators, and reciprocal relationships [e.g., Patient Assessment of Chronic Illness Care (PACIC), dyadic HF typology, and dyadic adjustment]. Additionally, health literacy, demographic information, spiritual and religious coping, and reciprocal relationships are measured for both participants and caregivers at baseline. Patient and caregiver outcome measures are described in detail in Table 1.

\section{Intervention}

The ENABLE CHF-PC intervention includes two major components: (1) an in-person comprehensive PCC as soon as feasible after enrollment (performed at the participants' study site, e.g., UAB or BVAMC supportive care clinic) and (2) telephone-based nurse coaching sessions following a six-session patient curriculum and a four-session caregiver curriculum followed by monthly supportive care check-in phone calls through the end of data collection (48 weeks) or patient death. The nurse coach interacts with the patient and FCG over time and across settings (home, clinic, hospital, and hospice) via telephone using the standardized curriculum and guidebook: Charting Your Course (CYC): An Intervention for Patients with Heart Failure and their Families (Fig. 2). The CYC materials are mailed to participants prior to the first session. Participants are asked to review each chapter prior to the session, but this is not required. Based on our prior work and recent pilot study [42, 54], sessions last approximately 30 to $40 \mathrm{~min}$. After notification of intervention allocation, the nurse coaches complete an introductory phone call to the patient or caregiver and confirm receipt of the CYC materials and schedule the first weekly session. The nurse coaches conduct the scheduled weekly sessions, directly entering field notes into the REDCap database, and then continue to follow participants monthly to check on patient and caregiver needs and to reinforce prior content.

PCC and nurse coach involvement is complementary; the in-person PCC provides expert symptom assessment and the nurse coaches' sessions deliver comprehensive palliative care and HF information in a convenient setting. The primary goal of the intervention is to 
Table 1 ENABLE CHF-PC randomized controlled trial outcome measures

\begin{tabular}{|c|c|c|c|}
\hline & Construct & Instrument & Description of measure \\
\hline \multicolumn{4}{|c|}{ Specific aim 1} \\
\hline \multirow[t]{5}{*}{$\begin{array}{l}\mathrm{HF} \\
\text { patients }\end{array}$} & $\mathrm{QOL}$ & $\begin{array}{l}\text { Kansas City Cardiomyopathy } \\
\text { Questionnaire (KCCQ) }\end{array}$ & $\begin{array}{l}5 \text { domains: physical limitations, } \\
\text { symptoms, self-efficacy, social } \\
\text { interference, and QOL; } 23 \text { items }\end{array}$ \\
\hline & Mood & $\begin{array}{l}\text { Hospital Anxiety and } \\
\text { Depression Scale (HADS) }\end{array}$ & $\begin{array}{l}2 \text { domains measuring depression } \\
\text { and anxiety; } 14 \text { items }\end{array}$ \\
\hline & $\begin{array}{l}\text { Symptom } \\
\text { burden }\end{array}$ & FACIT-Pal (14-item) & $\begin{array}{l}4 \text { domains: } \\
\text { physical, social/family, emotional, } \\
\text { and functional well-being; } 14 \text { items }\end{array}$ \\
\hline & & $\begin{array}{l}\text { PROMIS pain intensity scale; } \\
\text { 3-item SF and pain } \\
\text { interference ( } 2 \text { items) }\end{array}$ & $\begin{array}{l}\text { 3-item PROMIS pain intensity scale; } \\
3 \text { items; patient responses are from } \\
1 \text { (had no pain) to } 5 \text { (very severe); } \\
\text { PROMIS pain interference; } 2 \text { items }\end{array}$ \\
\hline & Resource use & Patient resource use & $\begin{array}{l}\text { Inpatient days, ICU days, ED visits, } \\
\text { hospice use, palliative care provider } \\
\text { visits, AD completion, DNR orders }\end{array}$ \\
\hline
\end{tabular}

Reliability Schedule
Subscales $a=0.62$ to 0.90 Baseline + every 8 weeks for 48 weeks

Subscales $a=0.82$ to 0.83

Subscales $a=0.75$ to 0.93

$a=0.33$ to 0.93 (intensity); NA (interfere)

NA
Specific aim 2

Caregivers QOL

Mood

Caregiver

burden

\section{HADS}

Montgomery Borgatta Caregiver Burden Scale

Positive Aspects of Caregiving (PAC)

Exploratory aims

HF Activation

Patient Assessment of Chronic Illness Care (PACIC)

Coping style Brief cope

Social support Multidimensional Scale of Perceived Social Support (MSPSS)

Health literacy Rapid Estimate of Adult Literacy in Medicine

Self-reported PROMIS SF Global Health health 10

Demographics Demographic questionnaire

Spiritual and

religious

Brief multidimensional measure of religiousness coping 15-item scale that measures changes in social functioning, subjective well-being, and somatic health of caregiver

(same as above)

Measure of caregiver burden with 3 domains: objective burden, stress burden, and demand burden; 14 items

Measure of caregiver's mental-affective state in relation to the caregiving experience; 9 items

5 dimensions: activation, delivery system/decision support, goal-setting problem-solving, and coordination; 20 items

2 subscales: active and avoidant coping; 28 items

Perceived adequacy of support from family and friends; 12 items

Measure of health literacy and numeracy based on ability to recognize common health-related terms; 7 items

2 domains: physical and mental health; 10 items

Age, gender, race, marital status, religion, education, occupation, health insurance, smoking, etc.

Assesses two patterns of religious and spiritual coping with stressful life events: positive religious and spiritual coping reflective of benevolent religious methods of understanding and dealing with life stressors; and negative religious and spiritual coping reflective of religious struggle in coping; 7 items
Subscales $a=0.62$ to 0.90 Baseline + every 8 weeks for 48 weeks

$a=0.68$ to 0.79

Baseline only

$a=0.81$

Baseline only

$a>0.80$

Baseline only

Subscales $a=0.81$ to 0.86 Baseline + every 8 weeks for 48 weeks

Baseline only

Subscale $a=0.83$

Baseline + every 8 weeks for 48 weeks 
Table 1 ENABLE CHF-PC randomized controlled trial outcome measures (Continued)

\begin{tabular}{|c|c|c|c|c|}
\hline Construct & Instrument & Description of measure & Reliability & Schedule \\
\hline \multirow[t]{2}{*}{$\begin{array}{l}\text { Reciprocal } \\
\text { relationships }\end{array}$} & Dyadic HF care typology & $\begin{array}{l}\text { Four basic categories of typology } \\
\text { for dyad }\end{array}$ & NA & Baseline only \\
\hline & $\begin{array}{l}\text { Dyadic adjustment } \\
\text { scale- SF }\end{array}$ & $\begin{array}{l}\text { Measures degree of agreement on } \\
\text { relational factors such as shared } \\
\text { philosophy, goals and time spent } \\
\text { together; } 7 \text { items }\end{array}$ & $\begin{array}{l}\text { Subscales for patients: } \\
a=0.70 ; \text { for caregivers: } \\
a=0.78\end{array}$ & $\begin{array}{l}\text { Baseline + every } \\
8 \text { weeks for } 48 \\
\text { weeks }\end{array}$ \\
\hline
\end{tabular}

QOL quality of life, BCOS Bakas Caregiving Outcomes Scale, NA not applicable, FACIT-Pal Functional Assessment of Chronic Illness Therapy: Palliative Care, HADS Hospital Anxiety and Depression Scale, HF heart failure, ICU intensive care unit, KCCQ Kansas City Cardiomyopathy Questionnaire, ED emergency department, $A D$ advance directive, DNR do not resuscitate, MSPSS Multidimensional Scale of Perceived Social Support, PAC Positive Aspects of Caregiving, PACIC Patient Assessment of Chronic IIIness Care, PROMIS Patient-Reported Health Outcome Measures Global Health, SF Short Form

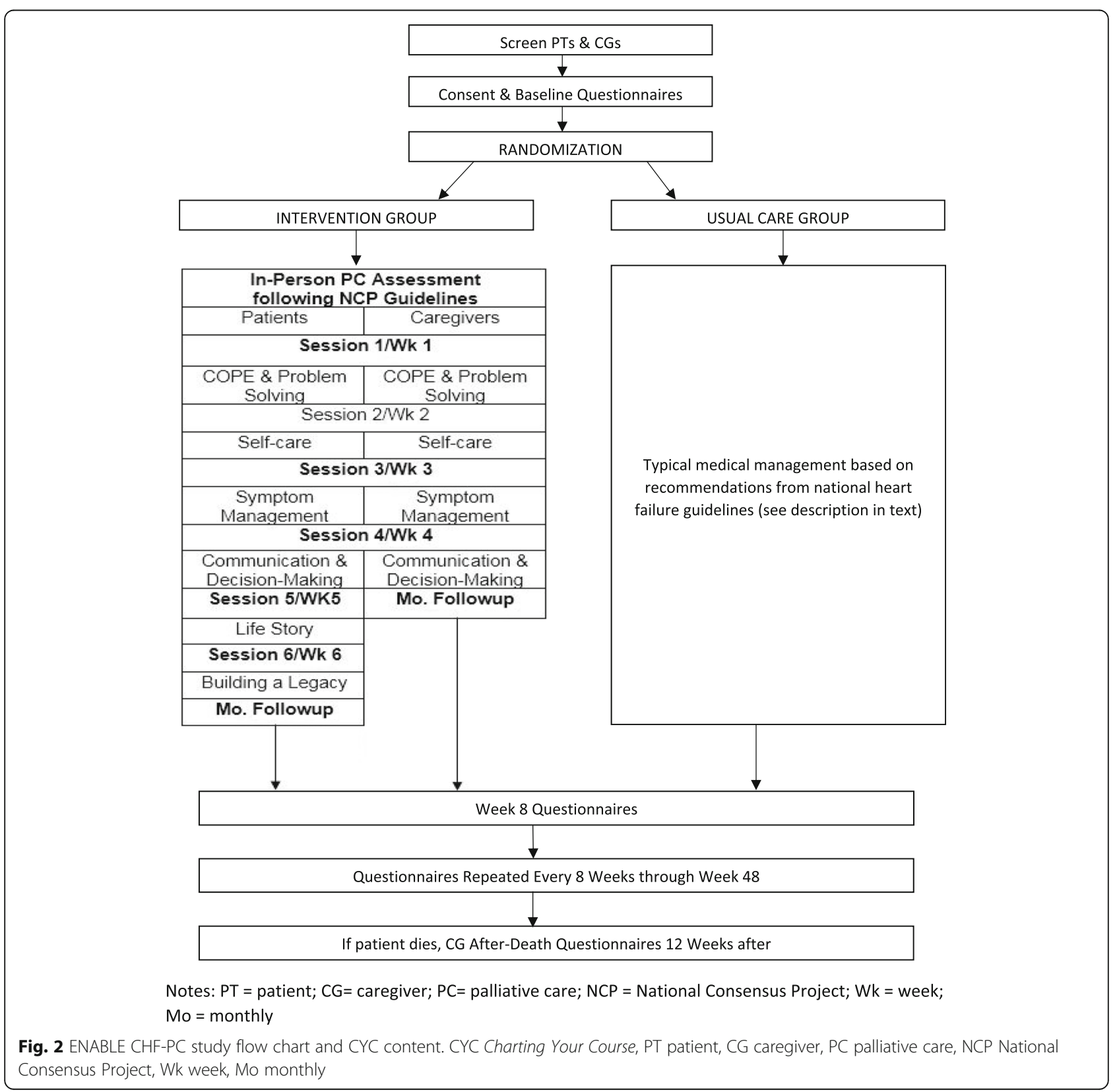


encourage patient empowerment; however, occasionally the nurse coaches may provide feedback directly to the HF teams (or palliative care teams) about specific issues (e.g., unrelieved pain) or makes referrals to other resources. The HF teams and palliative care teams are responsible for direct medical care. If an intervention patient is hospitalized, they will be followed by the attending service and a PCT consultation will be offered. Nurse coaches also work with participants to identify their local community resources or assist them in locating them, continuing to reinforce the community-based focus of the intervention.

\section{Usual care}

Patient participants randomized to the usual care group receive their cardiology-related outpatient care consistent with their care prior to enrolling in study. Typical HF patient medical management is based on national HF guidelines [10]. At UAB, HF patients randomized to the usual care group receive their outpatient care through the Advanced HF or Cardiology Clinics at the UAB Kirklin Clinic or the Cardiology Clinic located within UAB Hospital. At the BVAMC, HF patients are supervised by primary care, general cardiology, and geriatrics clinicians. Primary care practices are located in patients' local communities. Hospitalized patients are managed by the admitting service (cardiology or hospitalist). PCC are rare, but may be offered at the discretion of the clinician.

\section{Training and treatment fidelity monitoring}

To ensure fidelity of the intervention, we employ multiple strategies to address standardized provider training, measurement of treatment delivery, and evaluation of participant receipt. While experience of palliative care, cardiac, and psychiatric nursing was welcomed, it was not required for the nurse coach position. The nurse coaches are trained by the study staff, which includes $28 \mathrm{~h}$ of didactic and interactive role-play guided by an established training manual including specific intervention skills (e.g., problem-solving, decision support, and health coaching). Intervention experts review the digitally-recorded mock training sessions and provide feedback until the nurse coaches are confident in their skills. During intervention delivery, the nurse coaches enter field notes into the REDCap database in a template that corresponds to both the standardized script and to a previously developed fidelity checklist adapted to this study. Nurse coaches also participate in weekly meetings with experts to discuss ongoing intervention delivery experience and issues encountered during weekly participant calls. These weekly meetings also act as booster sessions. Expert external reviewers review 10\% of all digitally recorded coaching sessions, stratified by participant type, site, and nurse coach. The sessions are scored using fidelity checklists and nurse coaches are provided a copy of each scored session with both core component scores and an overall session score. Nurse coaches who exhibit a pattern of non-adherence $(<80 \%)$ on three consecutive ratings receive additional training and supervision. External reviewers review all PCC notes, which are recorded in the electronic medical record using a fidelity template comprising PCC elements based on National Consensus Project guidelines [58]. Clinicians completing the consultations are given aggregate feedback based on the template. Following the collection of outcome measures at week 48 , all intervention participants will be interviewed by phone to determine the extent of treatment receipt. These interviews are recorded digitally.

\section{Statistical analysis plan Specific aims}

Primary data analysis will begin by examining the balance between groups with descriptive statistics and measures of effect size for baseline characteristics and outcomes by treatment group. For patients, we will conduct longitudinal (intention-to-treat) analyses of the primary study outcomes (QOL and mood) for participants with baseline and one or more follow-up assessments using mixed effects modeling for repeated measures to examine the relative impact of ENABLE CHF-PC at 8 and 16 weeks after baseline. To be considered positive for patients and caregivers, statistically significant effects will need to be seen in patient QOL and mood and caregiver QOL, mood, and burden, respectively. Linear contrasts will be used to estimate the average post-intervention change for each group and for conducting tests of between-group differences. Standardized intervention effect sizes will be estimated for each outcome using baseline standard deviations. A false discovery rate criterion will be used to correct for multiple significance testing. Caregiver outcomes will be analyzed using the same longitudinal methods as outlined above. Only decedents' caregivers will have grief outcomes compared between groups, which will not involve longitudinal modeling.

\section{Exploratory aims}

Previously cited palliative care studies have shown alterations in both QOL and survival even though the interventions are not directly intended to impact survival. Therefore, we will compare differences in survival between groups using Kaplan-Meier plots and log-rank tests. Using path analytic models, we will examine whether the collected data support a hypothesized mediation mechanism in which the 
intervention effect on patient QOL, mood, and symptom burden is mediated by the quality of chronic illness care (PACIC), active and avoidant coping styles (COPE), social support (Multidimensional Scale of Perceived Social Support), and health literacy (Newest Vital Sign). All analyses will be performed using the latest versions of SAS and R.

\section{Sample size calculation}

We estimated the sample size for the specific aims assuming a time-averaged [64] between-group standardized minimum detectable difference of $d=.33$ (with five time-points, every 8 weeks from week 16 through 48 post-enrollment). Assuming an intra-subject correlation of 0.5 and a significance level of 0.01 , a sample size of 130 per group is necessary to achieve $80 \%$ power. After accounting for loss to follow-up of 30\% due to either death $(20 \%)$ or independent attrition $(10 \%)$, the target patient sample size was estimated at 190 per group (total $N_{\text {patient }}=380$ ) and 114 per caregiver group (total $N_{\text {caregiver }}=228$ ) with an overall participant total of 608 .

\section{Ethical aspects}

This study has been approved by the institutional review boards at UAB and BVAMC and registered on clinical trials.gov (NCT02505425). An independent data safety monitoring committee composed of UAB School of Nursing staff monitor the collection and analysis of data annually, including reviews of blind-protected preliminary analyses. All databases are secure, HIPPA-compliant, and password protected for both user front-end access and back-end storage. The back-end data is housed on a secure drive with access limited to only authorized research personnel. Study modifications are discussed with all investigators and research coordinators until consensus is reached. Amendments reflecting study modifications are submitted for institutional review board approval at all sites and updated on the clinical trial registry. The initial study protocol (version 1.0) was approved in 2014 and underwent minor modifications (version 1.1), which were approved in September 2015.

\section{Discussion}

Despite strong evidence for palliative treatments such as opioids for pain and dyspnea, psychotherapy, antidepressants, and multicomponent counseling interventions [30], few are part of routine HF care [10, 25]. There is growing evidence that providing palliative care early and concurrent with routine HF care can improve HF symptoms and QOL [10, 26, 38, 72], relieve physical and emotional suffering [19, 65-69], and possibly reduce hospitalizations [70-72]. However, there are currently few care models that consistently provide this care and little is known about age-dependent HF outcomes or treatment response because clinical exclusion criteria favor younger patients with less co-morbidity.

ENABLE CHF-PC is an innovative RCT that addresses the urgent need to integrate palliative care into the routine care of underserved older adults with HF in the following ways. First, ENABLE CHF-PC will be the first pragmatic RCT to study an extensively tested evidencebased telehealth palliative care coaching intervention tailored to older underserved adults. It introduces novel problem-solving, decision support techniques and patient decision aids, and life review early in the advancedHF trajectory as recommended by the 2013 ACC/AHA Guideline [15]. Second, ENABLE CHF-PC has a specific caregiver component, with a special focus on caregiver health literacy and self-reported health. Third, this RCT will contribute to palliative care science by examining factors that may impact patient and caregiver intervention mechanisms (e.g., health literacy, coping, and social support) and novel analytic methods that can inform future intervention development and testing. Finally, this multi-site study brings together an established interprofessional investigative team representing palliative care, HF, geriatrics, sociology, and behavioral psychology within a research-intensive infrastructure to address the special needs of underserved older adults with HF and their family caregivers.

Given the innovative nature of this early palliative care RCT for advanced-HF patients and caregivers, we have planned a multi-pronged approach to dissemination. Findings from this study will be disseminated through multiple peer-reviewed publications and presentations at national and international palliative care and cardiology conferences. Additionally, we plan to share our results through institutional websites and social media.

\section{Trial status}

ENABLE CHF-PC is an ongoing early palliative care RCT. Recruitment began in October 2015. At the time of submission of this manuscript, recruitment and randomization are actively ongoing and will continue through July 2018.

\section{Additional file}

Additional file 1: SPIRIT 2013 Checklist: Recommended items to address in a clinical trial protocol and related documents. (DOC $123 \mathrm{~kb}$ )

\section{Abbreviations}

ACC: American College of Cardiology; AD: Advance directive; AHA: American Heart Association; BCOS: Bakas Caregiving Outcomes Scale;

BVAMC: Birmingham Veterans Affairs Medical Center; CCM: Chronic Care Model; CG: Caregiver; CYC: Charting Your Course; DNR: Do not resuscitate; ED: Emergency department; ENABLE: Educate, Nurture, Advise, Before Life Ends; ENABLE CHF-PC: Educate, Nurture, Advise, Before Life Ends:

Comprehensive Heartcare for Patients and Caregivers; FACIT-Pal: Functional 
Assessment of Chronic Illness Therapy: Palliative Care; FCG: Family caregiver HADS: Hospital Anxiety and Depression Scale; HF: Heart failure; ICU: Intensive care unit; KCCQ: Kansas City Cardiomyopathy Questionnaire; Mo: Monthly; MSPSS: Multidimensional Scale of Perceived Social Support; NA: Not applicable; NCP: National Consensus Project; NIH: National Institutes of Health; NINR: National Institute of Nursing Research; NYHA: New York Heart Association; PAC: Positive Aspects of Caregiving; PACIC: Patient Assessment of Chronic Illness Care; PC: Palliative care; PCC: Palliative care consultation; PROMIS: Patient-Reported Health Outcome Measures Global Health; PT: Patient; QOL: Quality of life; RCT: Randomized controlled trial; REDCap: Research Electronic Data Capture; SPIRIT: Standard Protocol Items: Recommendation for Intervention Trials; UAB: University of Alabama at Birmingham

\section{Acknowledgments}

We would like to thank Karen Steinhauser for her invaluable contributions in developing the ENABLE intervention. We would like to thank all of the nurse coaches (Konda Keebler, Elizabeth Sockwell, and Sheri Tims) for their ongoing dedication in providing the intervention. We would like to thank all the clinicians and staff of the UAB Department of Cardiology (especially Renzo Loyaga-Rendon MD and Deepak Acharya MD), and the UAB Division of Geriatrics, Gerontology, and Palliative Care (especially Cathy Casey NP and Richard Taylor NP) for supporting the study. We would also like to thank Oladele Osasimi, Julie Schach, James Mapson, Diane Williams, Jacques DeBrow, Cynthia $Y$ Johnson, and Tawny Martin for assisting with recruitment and data collection.

\section{Funding}

This work was supported by the National Institute of Nursing Research (NINR) (R01NR013665). NINR did not have any role in study design, data collection, analysis, interpretation, or drafting the final reports of the study. JNDO receives support from the National Institutes of Health $(\mathrm{NIH})$ /National Institutes of Nursing Research (1K99NR015903). DE is supported by the NIH/NINR (3R01NR013665-02S1). RW is supported by the Robert Wood Johnson Foundation Future of Nursing Scholars Program. MS is supported by the University of Alabama at Birmingham Blazer Graduate Research Fellowship.

\section{Availability of data and materials}

The datasets that will be used and analyzed during the current study will be available from the corresponding author on reasonable request.

\section{Authors' contributions}

MS and RW were responsible for the main draft of the manuscript. MB conceived the study, oversees trial design, has overall responsibility for trial conduct, and provided substantial contributions to drafting the manuscript. JNDO assisted with study conception, oversees study conduct, and provided substantial contributions to drafting the manuscript. SVP, JT, EK, RT, KB, RD, and KMS assist with study design, conduct, patient referral, and treatment, and they reviewed the manuscript and provided critical feedback and a critique. AA developed the quantitative analysis protocol and reviewed and provided critical feedback of the manuscript. RW, DE, and SE assisted with protocol development and study conduct. RD and KS assisted with study conception and protocol development. All authors have given their approval of the final version of the manuscript.

\section{Ethics approval and consent to participate}

The study protocol was approved by the institutional review boards of the UAB (Birmingham, Alabama), and the BVAMC (Birmingham, Alabama). All patients and FCGs provided written informed consent.

\section{Consent for publication}

Not applicable.

\section{Competing interests}

The authors declare that they have no competing interests.

\section{Publisher's Note}

Springer Nature remains neutral with regard to jurisdictional claims in published maps and institutional affiliations.

\section{Author details}

School of Nursing, University of Alabama at Birmingham, 1720 2nd Avenue South, Birmingham, AL 35294-1210, USA. ²Department of Medicine, Division of Gerontology, Geriatrics, Palliative Care, University of Alabama at Birmingham, 1720 2nd Avenue South, Birmingham, AL 35294-1210, USA. ${ }^{3}$ Birmingham VA Medical Center, VAMC 11G, 700 19th St South, Birmingham, AL 35233-0001, USA. ${ }^{4}$ Department of Medicine, Division of Preventive Medicine, University of Alabama at Birmingham, 1720 2nd Avenue South, Birmingham, AL 35294-1210, USA. ${ }^{5}$ Department of Medicine, Division of Cardiovascular Diseases, University of Alabama at Birmingham, 1720 2nd Avenue South, Birmingham, AL 35294-1210, USA. ${ }^{6}$ Department of Medicine, Dell Medical School, University of Texas at Austin, 1501 Red River Street, Austin, TX 78712, USA. 'Department of Medicine, Division of Gerontology, Geriatrics, and Palliative Care, University of Alabama at Birmingham, 1720 2nd Avenue South, Birmingham, AL 35294-2041, USA.

Received: 7 March 2018 Accepted: 28 June 2018

Published online: 06 August 2018

\section{References}

1. Benjamin EJ, Virani SS, Callaway CW, Chang AR, Cheng S, Chuive SE, et al. Heart disease and stroke statistics_-2018 update: a report from the American Heart Association. Circulation. 2018; https://doi.org/10.1161/CIR. 0000000000000558 .

2. Liu L, Eisen HJ. Epidemiology of heart failure and scope of the problem. Cardiol Clin. 2014:32:1-8

3. Mozaffarian D, Benjamin EJ, Go AS, Arnett DK, Blaha MJ, Cushman M, et al. Executive summary: heart disease and stroke statistics_-2016 update. Circulation. 2016;133:447-54

4. Van Deursen VM, Damman K, Van der Meer P, Wijkstra PJ, Luijckx PJ, van Beck A, et al. Co-morbidities in heart failure. Heart Fail Rev. 2014;19(2):163-72.

5. Dionne-Odom JN, Hooker SA, Bekelman D, Ejem D, McGhan G, Kitko L, et al. Family caregiving for persons with heart failure at the intersection of heart failure and palliative care. Heart Fail Rev. 2017;22(5):543-57.

6. Jaarsma T, Beattie JM, Ryder M, Rutten T, McDonagh T, Mohacsi $P$, et al. Palliative care in heart failure: a position statement from the palliative care workshop of the heart failure Association of the European Society of cardiology. Eur J Heart Fail. 2009;11(5):433-43.

7. Allen LA, Stevenson LW, Grady KL, Goldstein NE, Matlock DD, Arnold RM, et al. Decision making in advanced heart failure. Circulation. 2012;125(15): 1928-52.

8. Feldman D, Pamboukian SV, Teuteberg JJ, Birks E, Leiz K, Moore SA, et al. The 2013 International Society for Heart and Lung Transplantation guidelines for mechanical circulatory support: executive summary. J Heart Lung Transplant. 2013;32(2):157-87.

9. Braun LT, Grady KL, Kutner JS, Adler E, Berlinger N, Boss R, et al. Palliative care and cardiovascular disease and stroke: a policy statement from the American Heart Association/American Stroke Association. Circulation. 2016; https://doi.org/10.1161/CIR.0000000000000438.

10. Yancy CW, Jessup M, Bozkurt B, Butler J, Casey DE Jr, Colvin MM, et al. 2017 ACC/AHA/HFSA Focused Update of the 2013 ACCF/AHA Guideline for the Management of Heart Failure: A Report of the American College of Cardiology/American Heart Association Task Force on Clinical Practice Guidelines and the Heart Failure Society of America. Circulation. 2017;23(8): 528-651.

11. Ponikowski P, Voors AA, Anker SD, Bueno H, Cleland JG, Coats AF, et al. 2016 ESC Guidelines for the diagnosis and treatment of acute and chronic heart failure: The Task Force for the diagnosis and treatment of acute and chronic heart failure of the European Society of Cardiology (ESC). Developed with the special contribution of the Heart Failure Association (HFA) of the ESC. Eur J Heart Fail. 2016;18(8):891-975.

12. Whellan DJ, Goodlin SJ, Dickinson MG, Heidenreich PA, Jaenicke C, Stough WG, et al. End-of-life care in patients with heart failure. J Card Fail. 2014; 20(2):121-34.

13. Meyers DE, Goodlin SJ. End-of-life decisions and palliative Care in Advanced Heart Failure. Can J Cardiol. 2016;32(9):1148-56.

14. Fang JC, Ewald GA, Allen LA, Butler J, Westlake Canary CA, Colvin-Adams M, et al. Advanced (stage D) heart failure: a statement from the Heart Failure Society of America guidelines committee. J Card Fail. 2015;21(6):519-34.

15. Yancy CW, Jessup M, Bozkurt B, Butler J, Casey DE Jr, Drazner MH, et al. 2013 ACCF/AHA Guideline for the Management of Heart Failure: A Report 
of the American College of Cardiology Foundation/American Heart Association Task Force on Practice Guidelines. J Am Coll Cardiol. 2013; 62(16):e147-239

16. World Health Organization. WHO Definition of Palliative Care. 2010. http:// www.who.int/cancer/palliative/definition/en/. Accessed Nov 2017.

17. Aldridge MD, Hasselaar J, Gerralda E, van der Eerden M, Stevenson D, McKendrick K, et al. Education, implementation, and policy barriers to greater integration of palliative care: a literature review. Palliat Med. 2016; https://doi.org/10.1177/0269216315606645.

18. Kavalieratos D, Gelfman LP, Tycon LE, Riegal B, Bekelman DB, et al. Palliative Care in Heart Failure: Rational, Evidence, and Future Priorities. J Am Coll Cardiol. 2017; https://doi.org/10.1016/j.jacc.2017.08.036.

19. Goodlin SJ. Palliative care in congestive heart failure. J Am Coll Cardiol. 2009;54(5):386-96.

20. Berry Jl. Hospice and heart disease: missed opportunities. J Pain Palliat Care Pharmacother. 2010;24(1):23-6.

21. Chattoo S, Atkin KM. Extending specialist palliative care to people with heart failure: semantic, historical and practical limitations to policy guidelines. Soc Sci Med. 2009;69(2):147-53.

22. Howlett JG. Palliative care in heart failure: addressing the largest care gap. Curr Opin Cardiol. 2011;26(2):144-8.

23. Hupcey JE, Penrod J, Fogg J. Heart failure and palliative care: implications in practice. J Palliat Med. 2009; https://doi.org/10.1089/jpm.2009.0010.

24. Beernaert K, Cohen J, Deliens L, Devroey D, Vanthomme K, Pardon K, Van den Block L. Referral to palliative care in COPD and other chronic diseases: a population-based study. Respir Med. 2013;107:1731-9.

25. Davis MP, Teml JS, Balboni T, Glare P. A review of the trials which examine early integration of outpatient and home palliative care for patients with serious illness. Ann Palliat Med. 2015; https://doi.org/10.3978/j.issn.22245820.2015.04.04

26. Janssen DJ, Spruit MA, Schols JM, Wouters EF. A call for high-quality advance care planning in outpatients with severe COPD or chronic heart failure. Chest. 2011; https://doi.org/10.1378/chest.10-1753.

27. Evangelista LS, Liao S, Motie M, De Michelis N, Lombardo D. On-going palliative care enhances perceived control and patient activation and reduces symptom distress in patients with symptomatic heart failure: a pilot study. Euro J Cardivasc Nurs. 2014; https://doi.org/10.1177/ 1474515114520766

28. Evangelista LS, Liao S, Motie M, De Michelis N, Ballard-Hernandez J, Lombardo D. Does the type and frequency of palliative care services received by patients with advanced heart failure impact symptom burden? J Palliat Med. 2014;17(1):75-9.

29. Evangelista LS, Lombardo D, Malik S, Ballard-Hernandez J, Motie M, Liao S. Examining the effects of an outpatient palliative care consultation on symptom burden, depression, and quality of life in patients with symptomatic heart failure. J Card Fail. 2012;18(12):894-9.

30. Bekelman DB, Nowels CT, Allen LA, Shakar S, Kutner JS, Matlock DD. Outpatient palliative care for chronic heart failure: a case series. J Palliat Med. 2011;14(7):815-21.

31. Kavalieratos D, Corbelli J, Zhang D, Dionne-Odom JN, Ernecoff NC, Hanmer J, et al. Association between palliative care and patient and caregiver outcomes: a systematic review and meta-analysis. JAMA. 2016;316(20):2104-14.

32. Fitzsimons D, Strachan PH. Overcoming the challenges of conducting research with people who have advanced heart failure and palliative care needs. Euro J Cardiovasc Nurs. 2011; https://doi.org/10.1016/j. ejcnurse.2010.12.002.

33. Bekelman DB, Nowels $C T$, Retrum JH, Allen LA, Shakar S, Hutt E, et al. Giving voice to patients' and family caregivers' needs in chronic heart failure: implications for palliative care programs. J Palliat Med. 2011;14(12):1317-24.

34. Thai V, Cujec B. Transitioning to end-of-life care for patients with advanced heart failure. J Palliat Med. 2010;13(7):796.

35. Davidson PM, Macdonald PS, Newton PJ, Currow DC. End stage heart failure patients - palliative care in general practice. Aust Fam Physician. 2010;39: 916-20.

36. Widera E, Pantilat SZ. Hospitalization as an opportunity to integrate palliative care in heart failure management. Curr Opin Support Palliat Care. 2009;3(4):247-51.

37. Bakitas M, Lyons K, Hegel MT, Balan S, Brokaw FC, Seville J, et al. Effects of a palliative care intervention on clinical outcomes in patients with advanced cancer: the project ENABLE II randomized controlled trial. JAMAv 2009; 302(7):741-749.
38. El-jawahri A, Greer JA, Temel JS. Does palliative care improve outcomes for patients with incurable illness? A review of the evidence. J Supp Oncol. 2011;9(3):87-94.

39. Temel JS, Greer JA, Muzikansky A, Gallegher ER, Admane S, Jackson VA, et al Early palliative care for patients with metastatic non-small-cell lung cancer. New Engl J Med. 2010;363(8):733-42.

40. Smith TJ, Temin S, Alesi ER, Abernethy AP, Balboni TA, Basch EM, et al. American Society of Clinical Oncology provisional clinical opinion: the integration of palliative care into standard oncology care. J Clin Oncol. 2012; 30:880-7.

41. Gomes B, Calanzani N, Curiale V, McCrone P, Higginson IJ. Effectiveness and cost-effectiveness of home palliative care services for adults with advanced illness and their caregivers. 2013; doi:https://doi.org/10.1002/14651858. CD007760.pub2.

42. Dionne-Odom JN, Kono A, Ahmed A, Frost J, Jackson L, Ellis D, et al. Translating and testing a concurrent palliative care model for rural-dwelling adults with heart failure and their family caregivers: ENABLE: CHF-PC. J Palliat Med. 2014; https://doi.org/10.1089/jpm.2013.0680.

43. Wagner EH. Chronic disease management: what will it take to improve care for chronic illness? Effect Clin Pract: ECP. 1998;1(1):2-4.

44. Wagner EH, Austin BT, Davis C, Hindmarsh M, Schaefer J, Bonomi A. Improving chronic illness care: translating evidence into action. Health Aff. 2001;20(6):64-78.

45. Bailey FA, Ferguson L, Williams BR, Woodby LL, Redden DT, Durham RM, et al. Palliative care intervention for choice and use of opioids in the last hours of life. J Gerontol A Biol Sci Med Sci. 2008;63(9):074-978.

46. Bailey FA, Burgio KL, Woodby LL, Williams BR, Redden DT, Kovac SH, et al. Improving processes of hospital care during the last hours of life. Arch Intern Med. 2005;165(15):1722-7.

47. Cheng JW. Nayar M. A review of heart failure management in the elderly population. Am J Geriatr Pharmacother. 2009;7(5):233-49.

48. Havranek EP, Masoudi FA, Westfall KA, Wolfe P, Ordin DL, Krumholz HM. Spectrum of heart failure in older patients: results from the National Heart Failure project. Am Heart J. 2002;143(3):412-7.

49. Martinez-Selles M, Vidan MT, Lopez-Palop R, Rexach L, Sánchez E, Datino T, et al. End-stage heart disease in the elderly. Rev Esp Cardiol. 2009;62(4):409-21.

50. Lorenz KA, Lynn J, Dy SM, Shurgerman LR, Wilkinson A, Mularshi RA, et al. Evidence for improving palliative care at the end of life: a systematic review. Ann Intern Med. 2008;148(2):147-59.

51. Zambroski CH, Moser DK, Roser LP, Heo S, Chung ML. Patients with heart failure who die in hospice. Am Heart J. 2005;149(3):558-64.

52. McAlister FA, Lawson FM, Teo KK, Armstrong PW. A systematic review of randomized trials of disease management programs in heart failure. Am J Med. 2001;110(5):378-84.

53. Hunt SA, Abraham WT, Chin MH, Feldmen AM, Francis GS, Graniats TC, et al. 2009 focused update incorporated into the ACC/AHA 2005 guidelines for the diagnosis and Management of Heart Failure in adults: a report of the American College of Cardiology Foundation/American Heart Association task force on practice guidelines: developed in collaboration with the International Society for Heart and Lung Transplantation. Circulation. 2009; 119(14):e391-479.

54. Bakitas M, Dionne-Odom JN, Pamboukian SV, Tallaj J, Kvale E, Swetz KM, et al. Engaging patients and families to create a feasible clinical trial integrating palliative and heart failure care: results of the ENABLE CHF-PC pilot clinical trial. BMC Palliat Care. 2017;16(1):45.

55. Steinhauser KE, Arnold RM, Olsen MK, Lindquist J, Hay J, Wood LL, et al. Comparing three life-limiting diseases: does diagnosis matter or is sick, sick? J Pain Symptom Manag. 2011; https://doi.org/10.1016/j. jpainsymman.2010.11.006.

56. Faller H, Stork S, Schuler M, Schowalter M, Steinbüchel T, Ertl G, et al. Depression and disease severity as predictors of health-related quality of life in patients with chronic heart failure--a structural equation modeling approach. J Card Fail. 2009;15(4):286-292 e282. Lewis EF. End of life care in advanced heart failure. Current treatment options in cardiovascular medicine. 2011;13:79-89

57. Goebel JR, Doering LV, Evangelista LS, Nyamathi AM, Maliski SL, Asch SM, et al. A comparative study of pain in heart failure and non-heart failure veterans. J Card Fail. 2009:15:24-30.

58. National Consensus Project: Clinical Practice Guidelines for Quality Palliative Care (3rd edition). https://www.nationalcoalitionhpc.org/ncp-guidelines2013/. Accessed 8 March 2018. 
59. Chan A-W, Tetzlaff JM, Altman DG, Laupacis A, Gøtzsche PC, Krleža-Jerić K, Hróbjartsson A, Mann H, Dickersin K, Berlin J, Doré C, Parulekar W, Summerskill W, Groves T, Schulz K, Sox H, Rockhold FW, Rennie D, Moher D. SPIRIT 2013 statement: defining standard protocol items for clinical trials. Ann Intern Med. 2013;158:200-7.

60. Chan A-W, Tetzlaff JM, Gøtzsche PC, Altman DG, Mann H, Berlin J, Dickersin K, Hróbjartsson A, Schulz KF, Parulekar WR, Krleža-Jerić K, Laupacis A, Moher D. SPIRIT 2013 explanation and elaboration: guidance for protocols of clinical trials. BMJ. 2013; https://doi.org/10.1136/bmj.e7586.

61. American Heart Association: Classes of Heart Failure. http://www.heart.org/ HEARTORG/Conditions/HeartFailure/AboutHeartFailure/Classes-of-HeartFailure_UCM_306328_Article.jsp\#.WoblJ6jwY2w. Accessed 8 March 2018.

62. Callahan C, Unverzagt F, Hui S, Perkins A, Hendrie H. Six-item screener to identify cognitive impairment among potential research subjects. Med Care. 2002;40:771-81.

63. McCarthy EP, Burns RB, Davis RB, Phillips RS. Barriers to hospice care among older patients dying with lung and colorectal cancer. J Clin Oncol. 2003;21: 728-35.

64. Brown H, Prescott R. Applied Mixed Models in Medicine. 2nd ed. Chichester, West Sussex, England: John Wiley \& Sons Ltd; 2006.

65. Bekelman DB, Rumsfeld JS, Havranek EP, Yamashita TE, Hutt E, Gottlieb SH, et al. Symptom burden, depression, and spiritual wellbeing: a comparison of heart failure and advanced cancer patients. J Gen Intern Med. 2009; https:// doi.org/10.1007/s11606-009-0931-y.

66. Currow DC, Smith J, Davidson PM, Newton PJ, Agar MR, Abernethy AP. Do the trajectories of dyspnea differ in prevalence and intensity by diagnosis at the end of life? J Pain Symptom Manag. 2010; https://doi.org/10.1016/j. jpainsymman.2009.09.017.

67. Goebel JR, Doering LV, Lorenz KA, Maliski SL, Nyamathi AM, Evangelista LS Caring for special populations: total pain theory in advanced heart failure: applications to research and practice. Nurs Forum. 2009;44:175-85.

68. Goebel JR, Doering LV, Shugarman LR, Asch SM, Sherbourne CD, Lanto AB, et al. Heart failure: the hidden problem of pain. J Pain Symptom Manag. 2009;38:698-707.

69. O'Leary N, Murphy NF, O'Loughlin C, Tiernan E, McDonald K. A comparative study of the palliative care needs of heart failure and cancer patients. Eur J Heart Fail. 2009;11:406-12.

70. Fang J, Mensah GA, Croft JB, Keenan NL. Heart failure-related hospitalization in the U.S., 1979 to 2004. J Am Coll Cardiol. 2008; https://doi.org/10.1016/j. jacc.2008.03.061.

71. Unroe KT, Greiner MA, Hernandez AF, Whellan DJ, Kaul P, Schulman KA, et al. Resource use in the last 6 months of life among medicare beneficiaries with heart failure, 2000-2007. Arch Intern Med. 2011; https://doi.org/10. 1001/archinternmed.2010.371.

72. Xu Z, Chen L, Jin S, Yang B, Chen X, \& Wu Z. (2018). Effect of palliative care for patients with heart failure. Int Heart J. 2018;59:503-9. https://doi.org/10. 1536/ihj.17-289.

Ready to submit your research? Choose BMC and benefit from:

- fast, convenient online submission

- thorough peer review by experienced researchers in your field

- rapid publication on acceptance

- support for research data, including large and complex data types

- gold Open Access which fosters wider collaboration and increased citations

- maximum visibility for your research: over $100 \mathrm{M}$ website views per year

At BMC, research is always in progress.

Learn more biomedcentral.com/submissions 